\title{
Initial Presenting as Acute Vertigo Syndrome in Sepsis Associated Encephalopathy: A Retrospective Case Series
}

\author{
Ye T Zhou ${ }^{1}$ and Dao MTong ${ }^{2 *}$ \\ ${ }^{1}$ Department of General Surgery, Affiliated Shuyang People' Hospital, Xuzhou Medical University, Jiangsu, China \\ ${ }^{2}$ Department of Neurology, Affiliated Shuyang People' Hospital, Xuzhou Medical University, Jiangsu, China
}

Received: October 13, 2017; Accepted: November 02, 2017; Published: November 06, 2017

*Corresponding author: Dao-Ming Tong, Department of Neurology, Affiliated Shuyang People's Hospital, Xuzhou Medical University, Xuzhou, Yingbin Road, Shucheng, Jiangsu 223600, China, Tel: 05278999 0120;fax: 05271358 7583;E-mail: tongdaoming@163.com

\begin{abstract}
Objective: To investigate whether sepsis associated encephalopathy (SAE) is a cause of acute vertigo syndrome (AVS).

Methods: From Jen 2014 to Des 2016 period, the clinical data in 7 patients who were from a hospital intensive care unit (ICU), because of misdiagnosed SAE whose complaint was vertigo or dizziness, was retrospectively analyzed.

Results: Among 7 misdiagnosed SAE patients, 5 were male and 2 were female, with a median age of 60 years (range, 23-82 years). All patients presented with AVS at onset. Of them, transient or recurrent AVS occurred in 6 patients, and persistent AVS in 1case. 3 patients by brain angiography showed the ischemic lesions in the central vestibular partway, however, all of 7 cases with AVS presented a central vestibular impaired mechanism from septic shock or sepsis, supporting AVS was a central AVS. The patient 1 only presented isolated central AVS and with no others brain dysfunction, so met the diagnosis of mild SAE, and with a favorable prognosis. The other 6 cases developed from mild brain dysfunction (central AVS) to deep coma and multiple organ failure, met the diagnosis of severe SAE with multiple organ failure, and all of them died eventually.
\end{abstract}

Conclusions: SAE might be a little-known cause of the central AVS. A recognition of this issue facilitate earlier identification and more timely treatment in patients with AVS with SAE.

\section{Introduction}

Acute vertigo syndrome (AVS) is also called acute vestibular syndrome (AVS). The AVS caused by the central vestibular pathways lesion is known as central AVS. There are many reasons for central AVS. The most common causes are acute ischemic stroke [1,2], followed by spontaneous intra cerebral hemorrhage $[1,3]$. Perhaps, there are many other well-known reasons for the first symptom that commonly precipitate central AVS, such as migraineous vertigo [4], heart disease [5], acute drug poisoning or adverse reactions (including carbon monoxide, phenytoin, antihypertensive drugs and antitumor drugs) [6-9], vertigo epilepsy[10], and multiple sclerosis [11]. In addition, rare causes may include hypoglycemia, acute leukemia, brain tumors, Hunter's bow syndrome, and potential malnutrition or anemia
[12-16]. In some cases, even smoking and drinking may also cause this syndrome. However, there are few reports of sepsis associated encephalopathy (SAE) with AVS as the initial symptom. Here, we retrospectively reviewed a series of clinical data from 7 consecutive SAE patients with AVS as the initial symptom, so as to improve the clinical workers' ability to recognize SAE early.

\section{Methods}

\section{Study settings}

This study was a retrospective case study of all registered patients from an adult intensive care unit (ICU) in China. A total of 7 SAE patients who had acute vertigo or dizziness as an initial symptom were recruited from January 2014 and December 2016. The Ethical Committee on Clinical Research of the Shuyang People's Hospital, China, approved the study. The study was in full compliance with the Helsinki declaration, and written informed consent was obtained from patients or their families.

\section{Patients and selection criteria}

We identified acute vertigo syndrome (H81.9) and central vertigo (H81.4) according to the master code of clinical revision (ICD-10-CM), Tenth Edition of the international classification of diseases of the WHO. We also treat sepsis and septic shock (A41.9) as the primary code for identifying sepsis events. Subjects included criteria: (1) patients with AVS who were the first symptom (over 18 years of age); (2) present evidence of a confirmed or suspected infection, and presence of sepsis or septic shock. Exclusion criteria: 1) less than 18 years old; 2) non septic or septic shock patients.

All data were extracted from electronic medical records. The following data in patients with septic shock initial presenting as AVS were recorded, such as age, gender, onset to admission time, body temperature, blood pressure, heart rate, respiratory rate, the general characteristics of acute dizziness syndrome, GCS score, PaO2, creatinine, bilirubin, serum glucose, lactate levels, white blood count, platelet count, bacteriological findings, ECG, and brain CT scans. We also recorded the findings of CT, including 
the location and size of the lesion.

The sequential organ failure score (SOFA) after AVS were recorded. We calculated the worst SOFA score during hospitalization. Finally, we recorded the number of days of ICU stay and hospitalization, and the prognosis after 30 days of follow-up. Survival and functional outcomes were assessed using a GOS score.

\section{Relation definitions}

The definition of sepsis has been updated by sepsis-3 in 2016 $[17,18]$, which was defined as a life-threatening organ dysfunction due to a dysregulated host response to infection. Septic shock is a specific form of sepsis in which profound circulatory, cellular and metabolic abnormalities are associated with a greater risk of mortality than with sepsis alone. In the absence of other causes for hypotension, the septic shock can be diagnosed as the patient's a systolic blood pressure reduction of $40 \mathrm{mmHg}$ from baseline, or the mean arterial pressure $<70 \mathrm{mmHg}$ and need to use vasopressors maintain, while serum lactic acid level $\geq 2 \mathrm{mmol} / \mathrm{L}$.

We are using the following criteria for the diagnosis of SAE: (1) Eligible diagnostic criteria for Sepsis-3; (2) present evidence of diffuse or multifocal cerebral dysfunction (from mental or behavioral changes to severe lethargy or coma); (3) brain imaging (CT or MRI) reveal the cortical and subcortical white matter ischemic lesions or micro infarction, or no obvious abnormality. The exclusion criteria were as follows: (1) presence of meningitis/ encephalitis or other encephalopathy; (2) present evidence of non sepsis or non septic shock.

Acute vestibular syndrome (AVS) is mainly characterized by acute vertigo or dizziness [19]. The AVS caused by impaired central vestibular pathways is called central AVS. The following criteria were used to diagnose central AVS: (1) sudden onset of vertigo or dizziness accompanied by head-motion intolerance, gait unsteadiness, nausea or vomiting, or nystagmus. (2) Patients with risk factors or pathogenesis of impaired central vestibular pathways; (3) present evidence of imaging or laboratory in central vestibular pathways lesion. In terms of symptomatology, central AVS is a manifestation of mental and neurological abnormity, i.e., mild brain dysfunction. Vertigo is described as a feeling of the rotation of oneself/space when human brain process un imbalance; severe cases may present with head-motion intolerance or abnormal balancing behavior, such as standing instability, even the whole itself rolled sharply to one side and fell to the floor. Dizziness is a top-heavy feeling, or also with a minor abnormal balancing behavior, including inclining and shaking. Dizziness or vertigo is usually transient (lasting seconds to minutes, generally $<24$ hours), but may occur in continuous repeated episodes, or persistent episodes, lasting more than a few days.

\section{Statistical methods}

The numeric variables were expressed as means \pm standard deviation (SD) or medians (IQR), and n (\%) for qualitative values. Fisher's exact test and the Mann-Whitney $U$ test were used to examine the relationship between baseline patient variables. Continuous variables were compared using Student's t test. All p-values were 2-sided, and significance was set at $\mathrm{P}<0.05$. Statistical calculations were performed using a proprietary, computerized statistics package (SPSS 10.0).

\section{Result}

In this clinical case series, 36 patients were admitted for septic shock, of those, only $7(19.4 \%)$ patients who met the inclusion / exclusion criteria were enrolled The clinical features of 7 patients with AVS are shown in (table 1). Five patients were male and 2 female, the median age was 60 years (range, 23-80). All patients had sudden onset of dizziness or vertigo as an initial symptoms. Of them, 6 patients (85.7\%) had transient AVS (usually within several minutes), and only 1 (14.3\%) was persistent AVS (lasting $24 \mathrm{~h}$ ). The most common associated symptoms were nausea and vomiting, but no one with nystagmus. The shortest time interval from AVS onset to admission was 10 minutes, the longest was 10 days, and the mean \pm SD was $52.7 \pm 72.9$ hours.

Table 1: Characteristics of 7septic patients with initial presenting as AVS

\begin{tabular}{|c|c|c|c|c|c|}
\hline Sex & Age & Initial symptoms & Types & Persistent time & With symptoms \\
\hline 1. Female & 59 & Dizziness & Persistent & 24 helayed & Nausea, sweet \\
\hline 2. Male & 76 & Dizziness & Transient & Current $8 \mathrm{~d}$ & Fever \\
\hline 3. Male & 80 & Vertigo & Transient & Recurrent24hs & Nausea, vomit \\
\hline 4. Female & 60 & Dizziness & Transient & Recurrent3d & Nausea, vomit \\
\hline 5. Male & 23 & Vertigo & Transient & 10min & Vomit \\
\hline 6. Male & 71 & Vertigo & Transient & Chs & disequilibrium \\
\hline 7. Male & 82 & Vertigo & Transient & Recurrent 10d & Noma \\
\hline
\end{tabular}

AVS=acute vestibular syndrome or acute vertigo syndrome 


\section{Characteristics of septic shock}

All of the patients with septic shock recorded, $5(83.3 \%)$ reported severe hypotension on admission, and only 2 patients reported nosocomial hypotension. All patients showed the manifestations of SIRS ( $\geq 2$ criteria), while they had findings associated with acute organ dysfunctions. Five patients $(71.4 \%)$ had confirmed infection, and only 2(26.6\%) had suspected infection. The most common site of infection was the respiratory tract (57.1\%). According to the new criteria for sepsis in 2016, the clinical and laboratory features of 7 patients with septic shock with central AVS are shown in (table 2).

\section{Clinical and imaging features of SAE}

Seven patients with clinical features of SAE were shown in table 3. In the 7 patients, five patients had brain imaging performed, and the other 2 cases were no brain imaging ( 1 case and 5 cases). Patient 2 showed no abnormalities in head CT scan within 24 hours of onset. Cranial CT in the case 4 at first 24 hours showed an old ischemic lesion, but no new lesions occurred. In the case 3 , initial cranial CT showed a small per ventricular infarction, met ischemic lesions of the central vestibular pathways. In the patient 6 and 7, initially DWI showed cortical and subcortical lacunars infarcts, met ischemic changes in the central vestibular pathway. Especially in the patient 6, his head MRI-DWI show the symmetry acute ischemic lesions in the bilateral insular lobes and in the per ventricular area.

\section{Discussion}

The acute brain dysfunction caused by impaired central vestibular pathways in the human brain is called central AVS. Focal lesion in the cortical or subcortical as well as brainstem or cerebellum is the most common causes of central AVS [1$4,10,11,15]$, but brain ischemia, hypoxia, poisoning, metabolic disorder, and malnutrition is also the factors of brain dysfunction caused by the lesions of vestibular central pathways [ 5-9,12$14,16]$.

The current series of 7 patients presented the following features: (1) present evidence of a suspected or confirmed infection; (2) the blood pressure decreased $40 \mathrm{mmHg}$, mean arterial blood pressure less than $70 \mathrm{mmHg}$, and the serum lactic acid level $>2 \mathrm{mmol} / \mathrm{L}$; (3) the existence of at least one organ dysfunction. Therefore, the diagnosis of septic shock or sepsis was established. However, our 7 patients with SAE failed to be diagnosed early because of lack of understanding of central AVS caused by brain dysfunction.

Table 2: Characteristics of clinical and laboratory in7 patients with septic shock

\begin{tabular}{|c|c|c|c|c|c|c|c|c|c|}
\hline Case/sex/age & $\begin{array}{l}\text { Infection } \\
\text { source }\end{array}$ & $\begin{array}{c}\text { Blood } \\
\text { Pressure(mmHg) }\end{array}$ & $\begin{array}{c}\text { Organ } \\
\text { failure }\end{array}$ & $\begin{array}{c}\text { SIRS } \\
\text { criteria }\end{array}$ & $\begin{array}{l}\text { Lactate } \\
\text { mmol/l }\end{array}$ & GCS & SOFA & GOS & Initial diagnosis \\
\hline 1/female/59 & Left lower leg & $75 / 50$ & + & 2 & 2.1 & 15 & 3 & 5 & Septic shock \\
\hline $2 /$ male $/ 76$ & Lungs & $72 / 50$ & + & 4 & 3.7 & $15 \rightarrow 4$ & 6 & 1 & Septic shock \\
\hline $3 /$ male $/ 80$ & Lungs & $53 / 31$ & + & 2 & 9.1 & 4 & 8 & 1 & Septic shock \\
\hline 4/female/60 & Unknown & $80 / 50$ & + & 2 & 5.7 & 4 & 9 & 1 & Septic shock \\
\hline $5 /$ male $/ 23$ & $\begin{array}{c}\text { Upper } \\
\text { respiratory }\end{array}$ & $97 / 50$ & + & 4 & 11.5 & 4 & 9 & 1 & Septic shock \\
\hline $6 /$ male/71 & Unknown & $160 / 90 \rightarrow 120 / 60$ & + & 2 & 3.5 & $15 \rightarrow 4$ & 9 & 1 & $\begin{array}{c}\text { Sepsis/septic } \\
\text { shock }\end{array}$ \\
\hline 7/male/82 & lungs & $142 / 76 \rightarrow 70 / 40$ & + & 4 & 1.8 & $15 \rightarrow 8$ & 6 & 1 & $\begin{array}{l}\text { Sepsis/septic } \\
\text { shock }\end{array}$ \\
\hline
\end{tabular}

AVS=acute vestibular syndrome or acute vertigo syndrome; SIRS=systemic inflammatory response syndrome; SOFA= sequential organ failure assessment; GCS= Glasgow Coma Scale; GOS= Glasgow outcome Scale.

Table 3: Characteristics and outcome of brain dysfunction in 7 septic shock patients

\begin{tabular}{|c|c|c|c|c|}
\hline Case/Sex/age & Brain Dysfunction & Imaging & MoD & Eutcome \\
\hline $1 /$ Demale/59 & AVS & No examine & Cir, brain & Mnosis \\
\hline $2 /$ male/76 & AVS $\rightarrow$ coma & No abnormal & Cir, brain, lung & Death \\
\hline $3 /$ male/80 & AVS $\rightarrow$ coma & Lacunar infarcts & Cir, brain, lung & Death \\
\hline $4 /$ female/60 & AVS $\rightarrow$ coma & No abnomal & Cir, brain, lung, live & Death \\
\hline $5 /$ male/23 & AVS $\rightarrow$ coma & No examine & Cir, brain, lung & Death \\
\hline $6 /$ male/71 & AVS $\rightarrow$ coma & Lacunar infarcts & Cir, brain, lung, live & Death \\
\hline $7 /$ male/82 & AVS $\rightarrow$ coma & Lacunar infarcts & Cir, lung, brain & SAE+MOD \\
\hline
\end{tabular}

SAE=sepsis associated encephalopathy; AVS= acute vestibular syndrome or acute vertigo syndrome; MOD=multiple organ dysfunction; 
Acute brain dysfunction, if its caused by sepsis or septic shock, is called SAE. The incidence of SAE accounts for approximately $70 \%$ of sepsis [20]. In view of the above, the diagnosis of SAE depends mainly on whether the patient has sepsis or septic shock. That is, as long as sepsis or septic shock is diagnosed, 2/3 may be associated with acute brain dysfunction. According to document, the patients with minor SAE may show mental or behavioral changes, severe SAE patients may also be presented with non- convulsive seizures, lethargy, or even coma. Moreover, there was no clinical and laboratory evidence of direct infection of the central nervous system, or any other reasons can be identified encephalopathy [21,22]. In fact, SAE is not uncommon, which has brain dysfunction as an onset or an isolated manifestation, and its initial manifestation may be a mild mental or behavioral changes, including hallucinations, irritability, delirium, inappropriate behavior, and so forth [21,22].

Previous studies have suggested that severe hypotension or low cardiac output is a common cause of global ischemia [23,24], and the watershed infarcts or dizziness is usually encountered[5]. In our current series, all patients with SAE had septic shock, suggesting that the pathogenesis of initial AVS is associated with brain ischemia $[5,23,24]$.

To our knowledge, SAE with AVS presenting as initial symptoms is not described. Of the current 6 patients, only patient 1 was satisfied with the diagnosis of SAE with isolated central AVS. The other 6 patients progressed from an initial central AVS to subsequent coma and multiple organ dysfunction (MOD), met the diagnosis of SAE with MOD. Prior studies have shown that infection can increase the incidence of acute ischemic stroke risk [25]. Although only patient 3,6 and 7 on CT or DWI showed small acute infarction in the cortical and subcortical areas, all patients showed a suspected infection or confirmed infection and having acute organ failure. Then, this evidence strongly suggests that SAE might contribute to the occurrence of initial central AVS.

The image changes of SAE are common with minor infarction and white matter lesion [26], and microcirculatory disturbance is the main pathogenesis in experimental models of sepsis [27]. Our patients 3,6 and 7 had minor infarcts and white matter ischemia imaging changes, which accord with the pathological mechanism of a dysregulated host response to infection.

However, some SAE may have severe brain microcirculatory disturbances that cause extensive subcortical white matter lesions, or multifocal necrotizing encephalopathy [28]. In this situation, there is often a poor prognosis. Unfortunately, our patients did not review brain imaging later.

Currently, sepsis and septic shock is still the most prevalent critical diseases worldwide. The morbidity in ICU for septic patients is $10 \%$ [29], while the fatality rate of SAE is as high as $51.0-72.0 \%$ [30]. However, SAE as one of the rare cause of initial central AVS has not been reported. We think an identification of this is important for early diagnosis and early treatment of
SAE's underlying infection. It is also beneficial to reduce SAE misdiagnosis and reduce mortality.

\section{Acknowledgments}

This work is supported by a grant from the Medical Research Council, affiliated Shuyang People's Hospital, Xuzhou Medical University (Clinical Key Specialty Construction Project of Jiangsu Provence, 20160013)

\section{References}

1. Tamutzer AA, Berkowitz AL, Robinson KA, Hsieh YH, NewmanToker DE. Does my dizzy patient have a stroke? A systematic review of bedside diagnosis in acute vestibular syndrome. CMAJ. 2011;183(9):E571-E592. doi:10.1503/cmaj.100174

2. Saber Tehrani AS, Kattah JC, Mantokoudis G, Pula JH, Nair D, Blitz A, et al. Small strokes causing severe vertigo: frequency of false-negative MRIs and nonlacunar mechanisms. Neurology. 2014;83(2):169-173.

3. Kim JS, Lee JH, Lee MC. Small primary intracerebral hemorrhage. Clinical presentation of 28 cases. Stroke. 1994;25(7):1500-1506.

4. Neuhauser H, Leopold M, von Brevern M, Arnold G, Lempert T. The interrelations of migraine, vertigo, and migrainous vertigo. Neurology. 2001;56(4):436-441.

5. Finsterer J, Stöllberger C. Neurological complications of cardiac disease (heart brain disorders). Minerva Med. 2016;107(1):14-25.

6. Bernas S, Rzepeck J, Winnicka R, Kołaciński Z, Krakowiak A. Acute poisonings with carbon monoxide among the patients hospitalized in the Toxicology Unit during the period of 2006-2010. Przegl Lek. 2012;69(8):415-419.

7. Hwang WJ, Tsai JJ. Acute phenytoin intoxication: causes, symptoms, misdiagnoses, and outcomes. Kaohsiung J Med Sci. 2004;20(12):580585. doi:10.1016/S1607-551X(09)70262-1

8. Bonnet G. A multicenter study of doxazosin in the treatment of essential hypertension in France. American Heart Journal. 1991;121(1):335-40.

9. Vietor NO, George BJ. Oxaliplatin-induced hepatocellular injury and ototoxicity: a review of the literature and report of unusual side effects of a commonly used chemotherapeutic agent. J Oncol Pharm Pract. 2012;18(3):355-359. doi:10.1177/1078155212437 901

10. Kim DW, Sunwoo JS, Lee SK. Incidence and localizing value of vertigo and dizziness in patients with epilepsy: Video-EEG monitoring study. Epilepsy Res. 2016;126:102-105. doi:10.1016/j. eplepsyres.2016.07.002

11. Pula JH, Newman-Toker DE, Kattah JC. Multiple sclerosis as a cause of the acute vestibular syndrome. J Neurol. 2013;260(6):1649-1654.

12. Binder C, Bendtson I. Endocrine emergencies. Hypoglycaemia. Baillieres Clin Endocrinol Metab. 1992;6(1):23-39.

13. Cartwright RA, Darwin C, McKinney PA, et al. Acute myeloid leukemia in adults: a case-control study in Yorkshire. Leukemia. 1988;2(10):687690.

14. Gergont A, Biedroń A, Zajac A, Steczkowska M. Causes and diagnostics of dizziness of central origin in children. Przegl Lek. 2009;66(11):983987.

15. Go G, Hwang SH, Park IS, Park H. Rotational Vertebral Artery Compression: Bow Hunter's Syndrome. J Korean Neurosurg Soc. 2013;54 (3):243-245. 
16. Agrawal Y, Carey JP, Della Santina CC, Schubert MC, Minor LB. Disorders of Balance and Vestibular Function in US Adults: Data from the National Health and Nutrition Examination Survey, 2001-2004. Arch Intern Med. 2009;169(10):938-944. doi:10.1001/archinternmed.2009.66

17.Singer M, Deutschman CS, Seymour CW, Shankar-Hari M, Annane $D$, Bauer $\mathrm{M}$, et al. The Third International Consensus Definitions for Sepsis and Septic Shock (Sepsis-3). JAMA. 2016;315(8):801-810. doi:10.1001/jama.2016.0287

18. Shankar-HariM, PhillipsGS, LevyML, Seymour CW, Liu VX, Deutschman CS, et al. Developing a New Definition and Assessing New Clinical Criteria for Septic Shock: For the Third International Consensus Definitions for Sepsis and Septic Shock (Sepsis-3). JAMA. 2016; 315(8):775-787. doi:10.1001/jama.2016.0289

19. Hotson JR, Baloh RW. Acute vestibular syndrome. N Engl J med. 1998;339:680-685.

20. Bolton CF, Young GB, Zochodne DW. The neurological complications of sepsis. Ann Neurol. 1993;33(1):94-100.

21.Gofton TE, Young GB. Sepsis associated encephalopathy. Nat Rev Neurol. 2012;8(10):557-566.

22. Wilson JX, Young GB. Progress in clinical neurosciences: Sepsisassociated encephalopathy: evolving concepts. Can J Neurol Sci. 2003;30(2):98-105.

23. Arbelaez A, Castillo M, Mukherji SK. Diffusion- weighted MR imaging of global cerebral anoxia. AJNR Am J Neuroradiol. 1999;20(6):9991007.
24. Verbeet T. Cardiovascular aspects of vertigo. Rev Med Brux. 2002;23(4):A351-A355.

25. Berger B, Gumbinger C, Steiner T, Sykora M. Epidemiologic features, risk factors, and outcome of sepsis in stroke patients treated on a neurologic intensive care unit. J Crit Care. 2014;29(2):241-248. doi:10.1016/j.jcrc.2013.11.001

26. Polito A, Eischwald F, Maho AL, Polito A, Azabou E, Annane D, et al. Pattern of brain injury in the acute setting of human septic shock. Crit Care. 2013;17(5):R204. doi:10.1186/cc12899

27. Taccone FS, Su F, Pierrakos C, He X, James S, Dewitte O, et al. Cerebral microcirculation is impaired during sepsis: an experimental study. Crit Care. 2010;14(4):R140. doi:10.1186/cc9205

28. Sharshar T, Gray F, Poron F, Raphael JC, Gajdos P, Annane D. Multifocal necrutizing leukoencephalopathy in septic shock. Crit Care Med. 2002;30(10):2371-2375.

29. Angus DC, Linde-Zwirble WT, Lidicker J, Clermont G, Carcillo J, Pinsky MR. Epidemiology of severe sepsis in the United States: analysis of incidence, outcome, and associated cost of care. Crit Care Med. 2001,29(7):1303-1310.

30. Sasse KC, Nauenberg E, Long A, Anton B, Tucker HJ, Hu TW. Long-term survival after intensive care unit admission with sepsis. Crit Care Med. 1995;23(6):1040-1047. 